\title{
Mapeo electromagnético en el dominio de las frecuencias y uso de la tomografía de resistividad eléctrica en el sitio hispánico de San Carlos de Portobelo, Panamá
}

\author{
Alexis Mojica ${ }^{1 *}$, Patricia Cid $^{2}$ y Leonardo Casini ${ }^{3}$ \\ ${ }^{1}$ Laboratorio de Investigación en Ingeniería y Ciencias Aplicadas, Centro Experimental de Ingeniería, \\ Universidad Tecnológica de Panamá \\ ${ }^{2}$ Facultad de Arquitectura y Diseño, Universidad Católica Santa María la Antigua, Panamá \\ ${ }^{3}$ Centro de Investigaciones para la Conservación del Patrimonio Arquitectónico y Urbano, Panamá
}

* Autor para correspondencia. Email: alexis.mojica@utp.ac.pa

Recibido: 17 de marzo de 2020

Aceptado: 08 de abril de 2020

\section{Resumen}

El estudio sobre la defensa militar de San Carlos de Portobelo, su arquitectura fortificada y las técnicas constructivas empleadas por los ingenieros militares españoles durante las últimas décadas del siglo XVII es una colaboración de la Universidad Católica Santa María la Antigua para la Dirección Nacional de Patrimonio Histórico del Ministerio de Cultura de Panamá. En la búsqueda de profundizar en el conocimiento del bien histórico ante la problemática del complejo de fortificaciones de Portobelo considerado actualmente: "Patrimonio Mundial en Peligro", se solicitó a la Universidad Tecnológica de Panamá la realización de pruebas geofísicas puntualizadas en el sitio, con el interés de obtener información que contribuya a determinar características constructivas del sistema de estructuras murarias actuales e identificar la ubicación de posibles componentes arquitectónicos bajo tierra a ser analizados en una futura etapa de estudios arqueológicos.

En este trabajo se presentan los resultados de una primera fase de prospección de tipo electromagnética en el dominio de las frecuencias y de resistividad eléctrica 2D en el sitio fortificado de San Carlos de Portobelo, provincia de Colón. Esta estructura de tipo hispánica constituye un sitio arquitectónico y arqueológico de gran interés debido a su trascendencia histórica, al no haberse concluido por la Corona Española. Las jornadas geofísicas fueron desarrolladas en dos zonas específicas: la Puerta de Tierra - entrada Sur de la ciudad - y en el Baluarte de San Pedro. En la primera zona, el mapa de conductividad eléctrica aparente obtenida con la configuración dipolar vertical (150 
cm de rango de profundidad efectiva) no reveló anomalías ligadas a rasgos arqueológicos enterrados; sin embargo, el mapa obtenido en el rango de profundidad efectiva de $75 \mathrm{~cm}$ (configuración dipolar horizontal) presentó algunas anomalías de baja conductividad eléctrica (alta resistividad eléctrica) que podrían estarasociadas a posibles rasgos enterrados o suelo firme, con un bajo contenido de humedad. En esta misma zona, las tomografías de resistividad eléctrica 2D ofrecieron información valiosa referente a la profundidad de los muros que aun afloran en esta área circunscrita del sitio, y de igual forma, los cimientos ubicados en las entradas de la mencionada zona. Por su parte, en el Baluarte de San Pedro, se desarrollaron 3 tomografías de resistividad eléctrica obteniéndose información referente a la profundidad del muro; el resto de las anomalías de baja resistividad eléctrica se asocian a suelo con alto contenido de humedad.

Palabras claves: Prospección geofísica, San Carlos de Portobelo, prospección electromagnética EM38 , tomografía de resistividad eléctrica $2 \mathrm{D}$, arquitectura fortificada.

\begin{abstract}
The study of the military defense of San Carlos on Portobelo, its fortified architecture and the construction techniques used by Spanish military engineers during the last decades of the 17th century is a collaboration of the Santa Maria la Antigua Catholic University to the National Directorate of Historical Heritage of the Ministry of Culture of Panama. In search to study the knowledge of the historical asset deeply in the face of the problems of Portobelo fortification complex currently considered as "World Heritage in Danger", the Technological University of Panama was requested to carry out geophysical tests in specific areas at the site, with the purpose to obtain information that contribute to determinate constructive characteristics of the current wall structure system and to identify the location of possible architectural components underground to be analyzed in a future stage of archaeological studies.
\end{abstract}

This work presents the results of a first phase of electromagnetic prospecting type in the frequency domain and 2D electrical resistivity at the fortified site of San Carlos on Portobelo, Colon Province. This Hispanic-type structure constitutes an architectural and archaeological site of great interest due to its historical significance of not having been concluded by the Spanish Crown. The geophysical sessions were developed in two specific areas: the Puerta de Tierra Gateway - South entrance to the city - and the San Pedro Bastion. In the first zone, the apparent electrical conductivity map obtained with the vertical dipole configuration (150 cm effective depth range) revealed no abnormalities linked to buried archaeological features; although, the map obtained in the effective depth range of $75 \mathrm{~cm}$ (horizontal dipole configuration) presented some anomalies of low electrical conductivity (high electrical resistivity) which could be associated with possible buried features or firm soil, with a low moisture content. In the same zone, 2D electrical resistivity scans offered valuable information regarding the depth of the walls that still emerge in this circumscribed area of the site and in the same way, the foundations located at the entrances of the mentioned area. Instead, in the San Pedro Bastion, 3 electrical resistivity tomographies were developed obtaining information regarding the depth of the wall; the rest of the anomalies of low electrical resistivity are associated with soil of high moisture content.

Keywords: Geophysical prospecting, San Carlos de Portobelo, electromagnetic survey EM-38, 2D electrical resistivity tomography, fortified architecture. 


\section{Introducción}

San Carlos de Portobelo ubicado en el interior de la histórica ciudad, inició su construcción en las últimas décadas de la segunda mitad del siglo XVII con la intención de albergar al poblado ubicado a orillas de la bahía que habría de transferirse al interno del área de San Cristóbal y ser protegido por una muralla defensiva que lo habría circundado. El proyecto de la nueva ciudad dedicado a Carlos II de España, fue concebido bajo los principios de las ciudades fortificadas europeas de influencia de la época y por los parámetros constructivos enunciados en los tratados de arquitectura militar. El proyecto nunca se culminó, pero las estructuras que aún restan nos permiten observar distintas etapas en el proceso de edificación de estos tipos de fortificaciones.

A finales de enero de 2020, se llevó a cabo una primera campaña de estudios geofísicos en el sitio del Baluarte de San Pedro y de la Puerta de Tierra como parte inicial de una serie de estudios que pretenden analizar las técnicas de construcción de las estructuras en piedra existentes y que, en gran parte, aún restan en pie hasta el Baluarte del Duque. El equipo estuvo conformado por A. Mojica, doctor en geofísica aplicada con experiencia en sitios de interés cultural; P. Cid y L. Casini, arquitectos restauradores con conocimientos en la historia de las fortificaciones de Portobelo y encargados de la dirección del proyecto; y M. Linero B., doctora en arqueología de la arquitectura quien brindó su asesoría en esta etapa.

El empleo de las prospecciones geofísicas como herramienta de investigación científica aplicada a este proyecto, forma parte de la "fase de trabajo de campo" y ha permitido obtener importantes datos preliminares con los cuales, iniciar el proceso analítico de verificación in situ de la documentación histórica y de la caracterización técnica de las estructuras arquitectónicas, además de, contribuir a la determinación de áreas de interés para el proyecto y que se requieren sean analizadas próximamente a través de un estudio arqueológico.

La prospección geofísica es una disciplina científica relativamente nueva, que emplea las estructuras matemáticas que caracterizan algunos fenómenos de la naturaleza para determinar las propiedades físicas de las rocas o suelos y sus contrastes. La prospección geofísica se caracteriza por abarcar un conjunto de técnicas que se enfocan en obtener un conocimiento sobre la distribución espacial de las rocas, materiales y formaciones en el interior del planeta, siendo éstas de interés al ser humano, por ejemplo: las fuentes de agua subterránea, rasgos arqueológicos, mantos rocosos, fallas tectónicas y contaminantes.

Entre los métodos de prospección geofísica más empleados, caben mencionarse los eléctricos y electromagnéticos (enfocados al estudio de las propiedades eléctricas del subsuelo), los gravimétricos y magnéticos (los cuales se ocupan del estudio de la densidad y la susceptibilidad magnética de los materiales, respectivamente) y los sísmicos (focalizados en el estudio de las propiedades elásticas de los suelos). Existen otros métodos geofísicos que se utilizan, pero no con mucha frecuencia como los térmicos y radiactivos.

Exceptuando las técnicas de exploración magnética, los métodos electromagnéticos han jugado un rol importante en el campo de la minería; no obstante, sus aplicaciones no se han extendido de forma amplia a la resolución de problemas de ingeniería civil, pero han tenido un papel notable en la detección de estructuras metálicas enterradas (cables y tubos metálicos, por ejemplo). En el campo 
ambiental, se ha visto un auge significativo en el mapeo superficial de infiltraciones de contaminantes (Telford et al., 1996).

En Arqueología, sus métodos clásicos incluyen excavaciones sistemáticas que demandan un notable esfuerzo humano, recurso económico y tiempo; en este aspecto, los métodos de mapeo geofísico emergen como una alternativa de la rápida evaluación del potencial arqueológico de un determinado sitio, siendo los eléctricos y magnéticos los más empleados (Tabbagh, 1992); no obstante, un tercer método geofísico está siendo muy utilizado hoy día: el electromagnético en el dominio frecuencial.

Estas metodologías no invasivas vendrían a representar, por ende, una nueva herramienta opcional de apoyo a la actividad arqueológica. El incremento en el uso de técnicas geofísicas para resolver problemas de tipo arqueológico constituye un hecho que revelan el éxito en la preservación de la herencia histórica de muchas ciudades. Los métodos electromagnéticos fueron empleados inicialmente durante los años 60 del siglo pasado, pero no fue hasta finales de ese mismo siglo que su uso se incrementó en estudios de tipo arqueológico arqueológico y agrícola. Son varias las investigaciones que han sido realizadas con éxito empleando este tipo de tecnologías electromagnéticas en estudios arqueológicos, por ejemplo, Bevan (1983); Frohlich y Lancaster(1985); Wynn (1986); Scollar et al., (1990); Dalan (1991).

El objetivo de este trabajo se focaliza en detectar y parametrizar posibles rasgos arqueológicos que se encuentren ligados a los restos construidos de la muralla defensiva de San Carlos ubicada en las cercanías de las fortificaciones de la bahía de Portobelo, específicamente en dos zonas importantes, mediante el uso de los métodos electromagnético - en el dominio frecuencial- y tomográfico de resistividad eléctrica $2 \mathrm{D}$.

\section{El sitio}

El sitio de interés se encuentra ubicado en el corregimiento de Portobelo, distrito del mismo nombre. La Figura 1 presenta la localización del área.

El objetivo de la Corona Española era la de trasladarla antigua ciudad de Portobelo a una nueva ciudad amurallada con la capacidad de contrarrestar el ataque de los piratas. Las Figuras 2 y 3 presentan un mapa antiguo de dicha estructura que data de 1683 y una imagen aérea actual, respectivamente. 


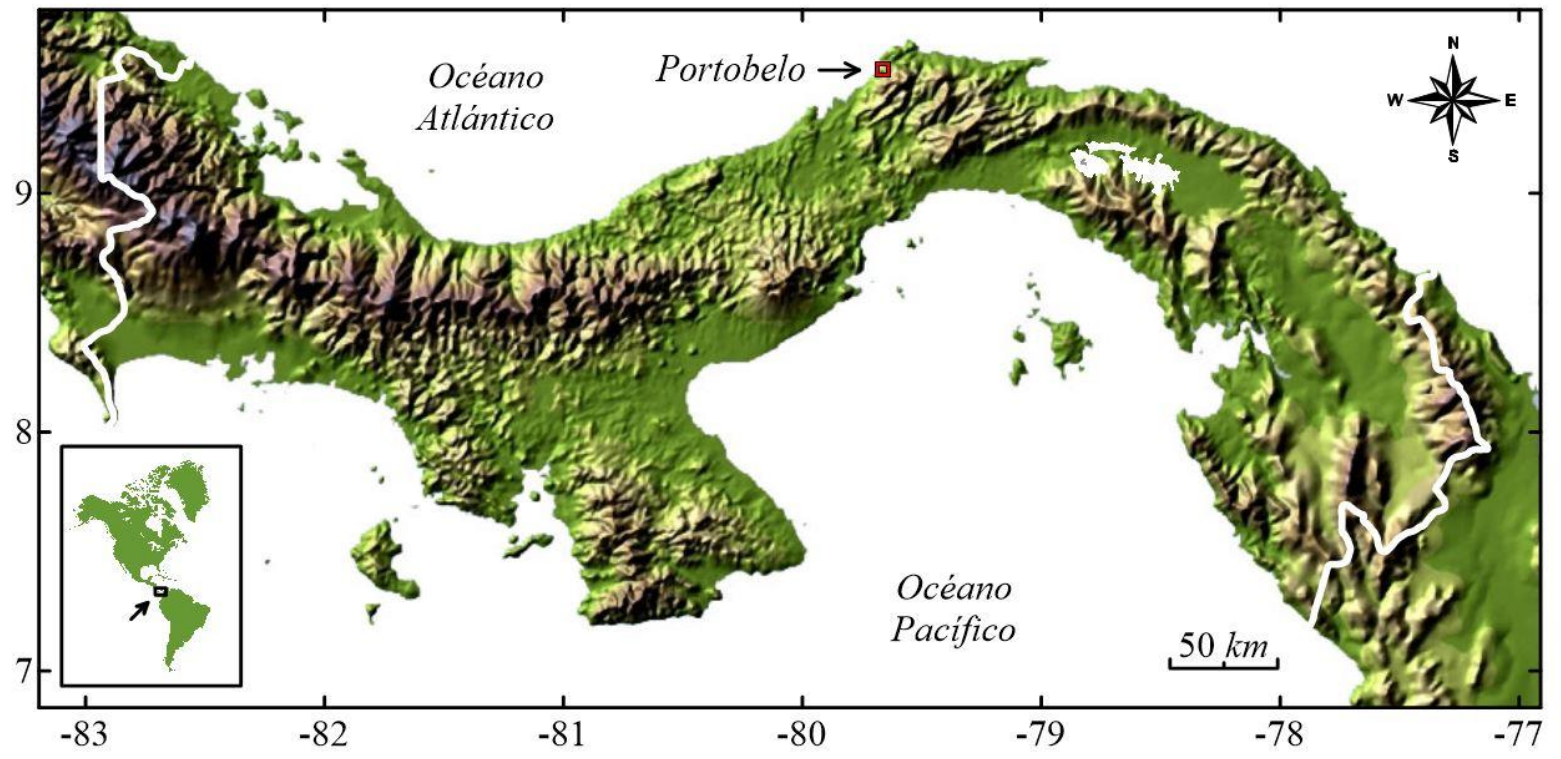

Figura 1. Localización geográfica de Portobelo, sector Norte del Istmo de Panamá.

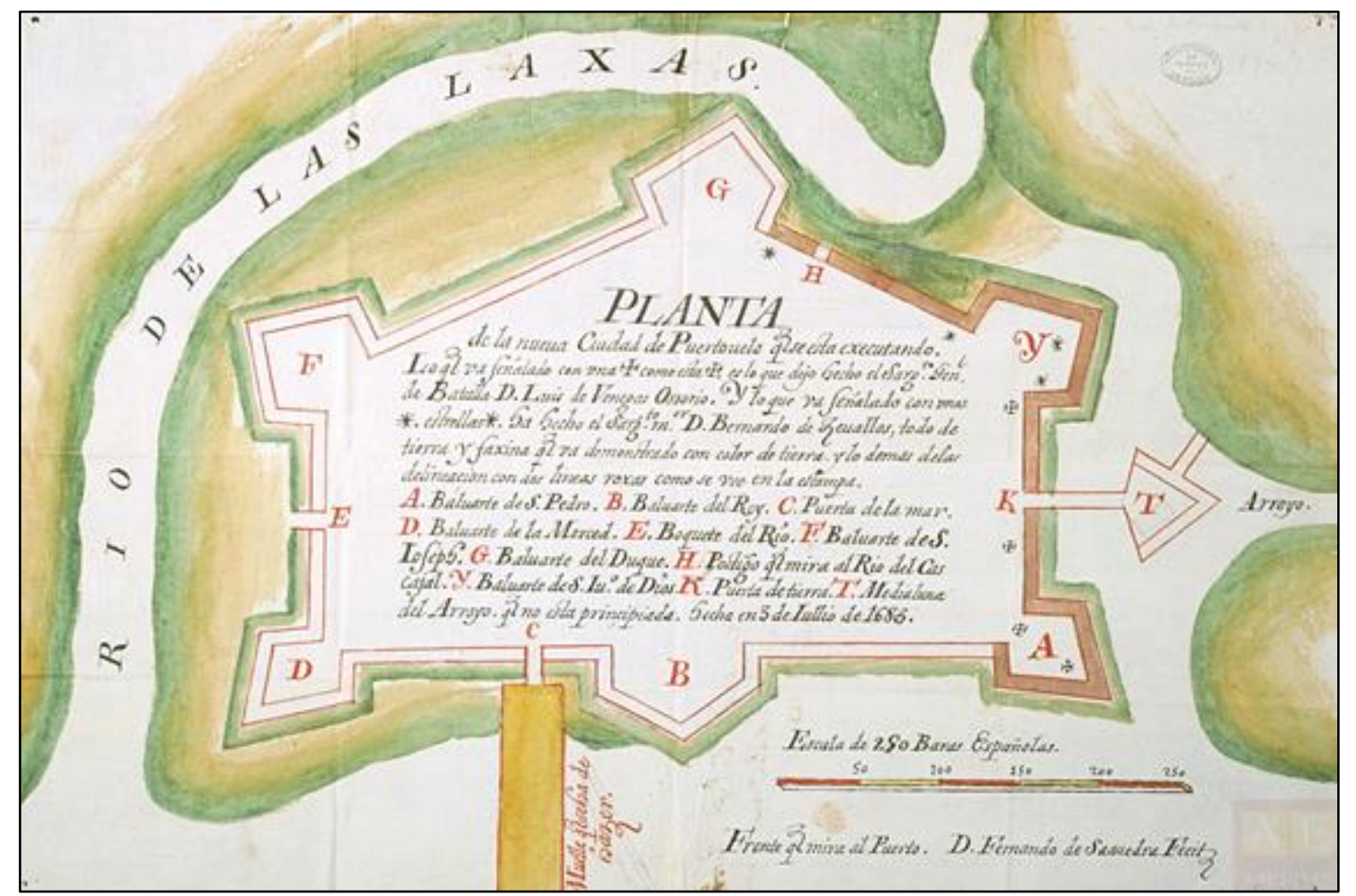

Figura 2. "Planta de la nueua Ciudad de Puertouelo que se está executando", Fernando de Saavedra, 1683. A - Baluarte de San Pedro. K - Puerta de Tierra. G - Baluarte delDuque. AGI (Archivo General de Indias),MP-PANAMA,95. 


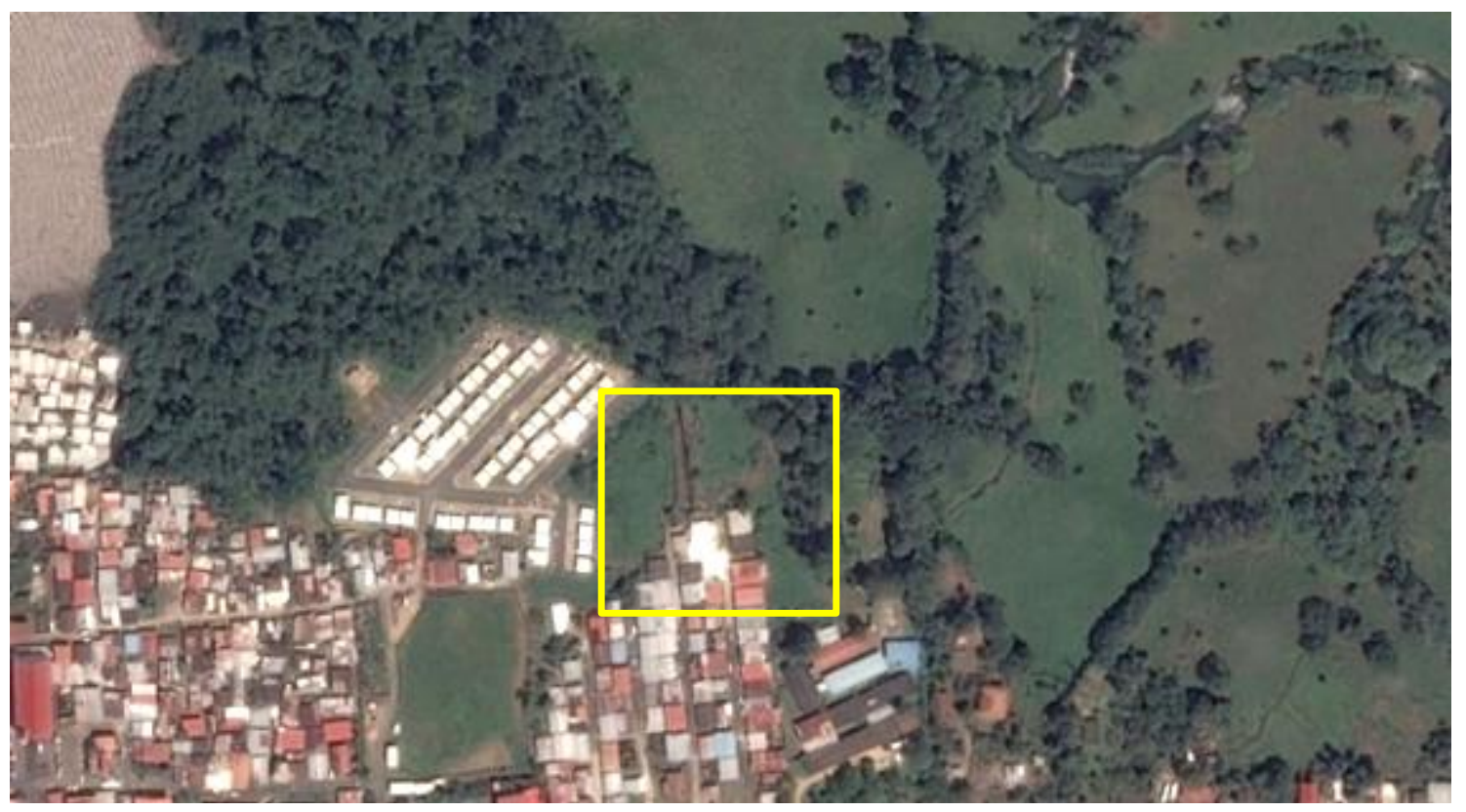

Figura 3. Imagen aérea que muestra los restos del Baluarte de San Pedro.

\section{La prospección geofísica}

En estudios arqueológicos, la detección de rasgos culturales se encuentra basada en la medición de una diferencia o contraste entre las propiedades físicas (conductividad eléctrica aparente o su inversa, la resistividad eléctrica aparente) de los materiales que componen dichos rasgos y del medio que la rodea, motivo por el cual se hace preponderante el estudio de las propiedades físicas de los suelos bajo las cuales los diferentes métodos geofísicos se encuentran basados (Scollar et al., 1990).

\subsection{La prospección electromagnética en el dominio frecuencial}

Los fenómenos electromagnéticos pueden ser descritos mediante las ecuaciones de Maxwell, las cuales describen la relación estrecha que existe entre los campos eléctrico y magnético y la interacción de estos con las estructuras o materiales contenidos en el subsuelo. El principio de operación de esta metodología se basa en el uso de una bobina emisora por donde circula una determinada intensidad de corriente eléctrica de tipo alterna; dicha circulación genera un campo magnético primario $\left(H_{p}\right)$ que oscila en los alrededores del punto de medición. Luego, este campo primario oscilante genera una variación en el flujo del campo magnético en el subsuelo, que a su vez produce corrientes secundarias inducidas en el mismo. Finalmente, estas corrientes secundarias crean en el interior de suelo un campo magnético secundario $\left(H_{S}\right)$ el cual es registrado en conjunto con el campo magnético primario por una bobina receptora. La razón de ambos campos registrados por el dispositivo EM-38 obedece a la siguiente ecuación:

$$
\sigma_{a}=\frac{2}{\pi \mu_{0} f} \frac{1}{L^{2}}\left[\frac{H_{s}}{H_{p}}\right]
$$


En donde $L$ representa la separación de las bobinas de emisión y recepción, $\mu_{0}$ la permeabilidad magnética del vacío y $f$ la frecuencia de oscilación. Para valores débiles de conductividad eléctrica $\sigma(<100 \mathrm{mS} / \mathrm{m})$, McNeill (1980) demostró que cuando un determinado dispositivo de inducción electromagnética (tipo Slingram EM-38, por ejemplo) se ubica en la superficie del suelo, la medición de $\sigma_{a}$ (conductividad eléctrica aparente) está dada por la ecuación anterior. Con respecto a la respuesta instrumental en función de la profundidad, es posible construir dos funciones $\Phi_{V}(z)$ y $\Phi_{H}(z)$ que describen las contribuciones relativas del campo magnético secundario que se deriva de una capa delgada a cualquier profundidad $z$ tanto para dos configuraciones dipolares vertical y horizontal, respectivamente. La Figura 4 muestra una representación gráfica de estas contribuciones.

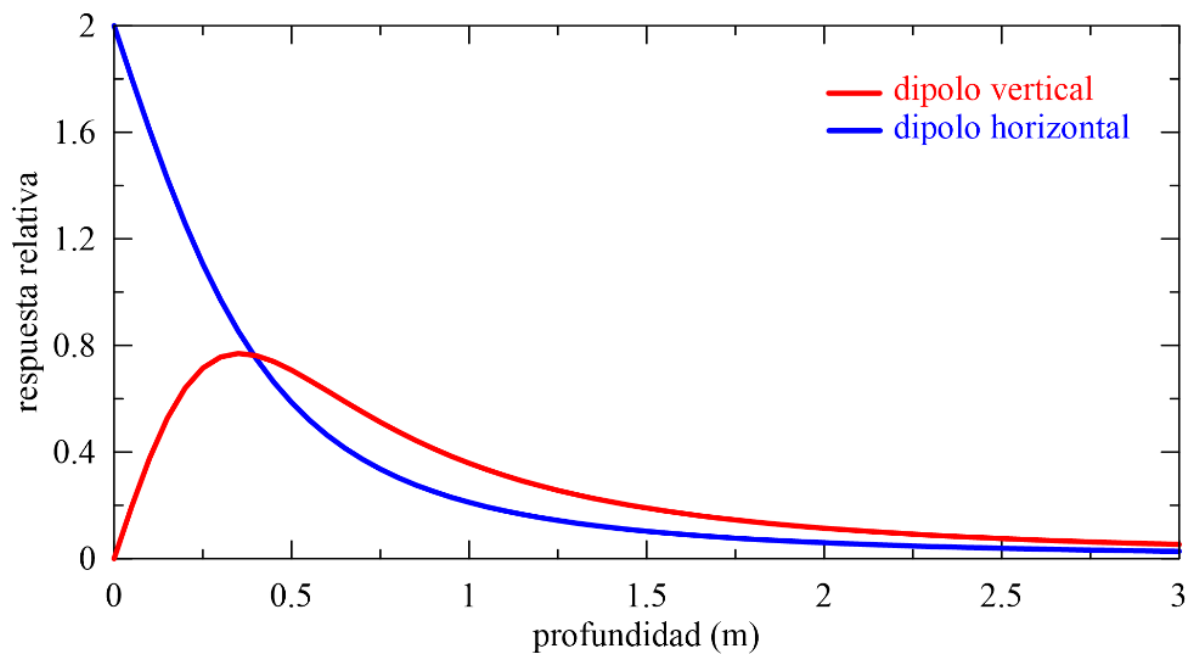

Figura 4. Comparación de las respuestas relativas de los dipolos horizontal y vertical.

En los últimos años, la utilización de dispositivos electromagnéticos tipo Slingram o EM-38 en estudios arqueológicos ha sido notable; este tipo de instrumentos emplea la inducción electromagnética con el objetivo de medir el valor de la conductividad eléctrica aparente de una columna de suelo hasta una profundidad de observación específica (Sudduth et al., 2001). La Figura 5 presenta una panorámica de este tipo de dispositivos los cuales son distribuidos por Geonics Inc. (Canadá).

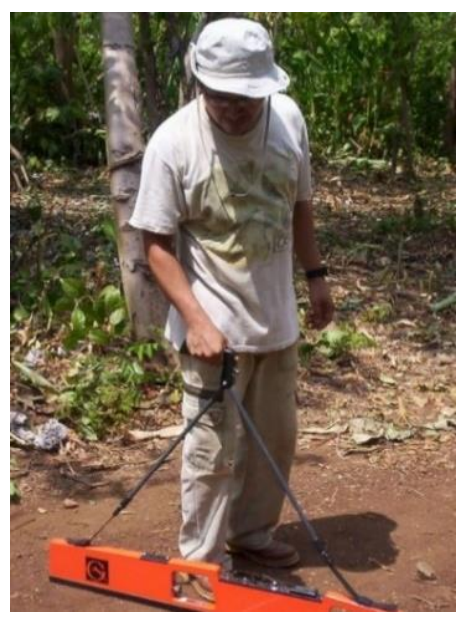

Figura 5. Dispositivo EM-38 de Geonics Inc. utilizado para la medición de la conductividad eléctrica aparente del subsuelo en modo dipolarvertical. 
En este tipo de dispositivos electromagnéticos, las ecuaciones matemáticas que relacionan las respuestas relativas con respecto a $z$ son:

$$
\Phi_{H}(z)=2-\frac{4 z}{\sqrt{4 z^{2}+1}} \quad y \quad \Phi_{V}(z)=\frac{4 z}{\sqrt{\left(4 z^{2}+1\right)^{3}}}
$$

\subsection{La tomografía de resistividad eléctrica $2 \mathrm{D}$}

En esta metodología, las propiedades eléctricas de las rocas y los minerales se encuentran controladas por diversos procesos químicos y por los parámetros termodinámicos como por ejemplo la presión y la temperatura del medio en el cual las cargas eléctricas se mueven (Nover, 2005); la movilidad que experimentan estas cargas la gobierna cuatro procesos diferentes de transporte: la conducción electrolítica de los fluidos contenidos en las rocas porosas saturadas y que depende de sus propiedades petrofísicas, la conducción electrónica que al igual que la electrolítica dependen de forma moderada de la temperatura y la presión, la semi-conducción que se da lugar cuando la temperatura de los minerales que forman las rocas aumenta y debido a la mayor movilidad de los defectos en la red y las impurezas, y la fusión parcial la cual aumenta la conductividad eléctrica si las temperaturas son muy elevadas.

El parámetro de interés en este tipo de análisis es la resistividad aparente la cual se expresa en ohm.m y representa el grado de dificultad que experimentan las cargas eléctricas al atravesar un determinado volumen de terreno. A parte de las características termodinámicas mencionadas con anterioridad, este parámetro depende de una serie de propiedades del suelo: la naturaleza de los sólidos que la constituyen como la distribución del tamaño de las partículas y mineralogía, el arreglo de los vacíos como la porosidad, la distribución del tamaño de los poros y la conectividad, el grado de saturación del agua o contenido de agua y la resistividad eléctrica de los fluidos (Samoüelian et al, 2005; Nover, 2005). Esta técnica de exploración geofísica se caracteriza por la inyección de una corriente eléctrica en el subsuelo a través de dos electrodos $(A$ y $B)$ y la medición de la diferencia de potencial mediante otro par de electrodos $(M$ y $N)$ incrustados en el suelo. Si en un punto sobre la superficie de interés se conocen estos dos parámetros y la geometría del arreglo electródico utilizado, entonces es posible calcular la resistividad eléctrica aparente del subsuelo.

Para la realización de estas pruebas, se utilizó un resistivímetro Syscal R1 (de IRIS Instruments). Dicha medición es representativa de un determinado volumen de suelo que depende de la posición y de la geometría de los electrodos utilizados; esto signific a que existe una gama de arreglos electródicos que se utilizan de acuerdo con la necesidad del trabajo a realizar. Debido al bajo nivel de ruido telúrico que presenta el sitio y la débil profundidad de exploración a investigar $(<3 \mathrm{~m})$, se empleó una configu ración electródica tipo dipolo - dipolo, en donde la distancia $A B=M N=a$, y este par se encuentra separado una distancia an, siendo $n$ un valor entero $(1,2,3,4,5)$; el segundo arreglo electródico empleado en este trabajo fue el Wenner-alfa, en donde las distancias $A M=M N=N B=l$ siendo el mismo poco sensible al ruido telúrico y con una profundidad de investigación superior al dipolo - dipolo. Para obtener una imagen bidimensional del subsuelo en términos de sus propiedades eléctricas, se necesita recurrir a complejos conceptos matemáticos relacionados con la resolución del problema inverso. Esta teoría se focaliza en determinar un modelo que ofrezca una respuesta del modelo (conjunto de valores de resistividad eléctrica aparente sintéticos) similar a los datos de resistividad aparente medidos sobre la superficie del área de interés. Este modelo, obtenido luego de un proceso iterativo, corresponderá 
a la distribución de la resistividad eléctrica real del subsuelo; por otro lado, la respuesta del modelo se obtiene a partir de las ecuaciones físicas que ligan el fenómeno con el modelo. Para caracterizar este modelo, se necesita discretizar el subsuelo a través de un conjunto de elementos cúbicos rectangulares o prismas, y los valores de resistividad eléctrica de dichos elementos corresponderán a los parámetros del modelo. Este cálculo se realiza a través de la resolución del problema directo, en donde se busca resolver una ecuación diferencial de segundo orden y cuya estructura matemática se obtiene de las leyes de conservación en Física y la ecuación de continuidad. Dicha ecuación tiene la forma:

$$
-\nabla\left[\frac{1}{\rho(x, y, z)} \nabla V(x, y, z)\right]=I \delta\left(x_{S}\right) \delta\left(y_{S}\right) \delta\left(z_{S}\right)
$$

Donde $\rho(x, y, z)$ es la distribución de la resistividad eléctrica en el espacio tridimensional, $I$ es la intensidad de la corriente ubicada en el punto $\left(x_{S}, y_{S}, z_{S}\right), \delta$ corresponde a la función delta de Dirac y $V(x, y, z)$ es la distribución del potencial eléctrico y su solución se obtiene derivando la ecuación anterior mediante una discretización del espacio $(x, y)$ bajo el cual el problema es resuelto. El algoritmo de modelado 2D el cual se basa en una aproximación de diferencia finita, utiliza la geometría de malla tridimensional establecida por Dey y Morrison (1979).

\section{Metodología}

\subsection{El mapeo electromagnético EM-38}

En el sector de la Puerta de Tierra, entrada Sur del sitio de San Carlos, se definió un rectángulo de 14 $\mathrm{m} \times 9 \mathrm{~m}$ tal como se muestra en la Figura 6.

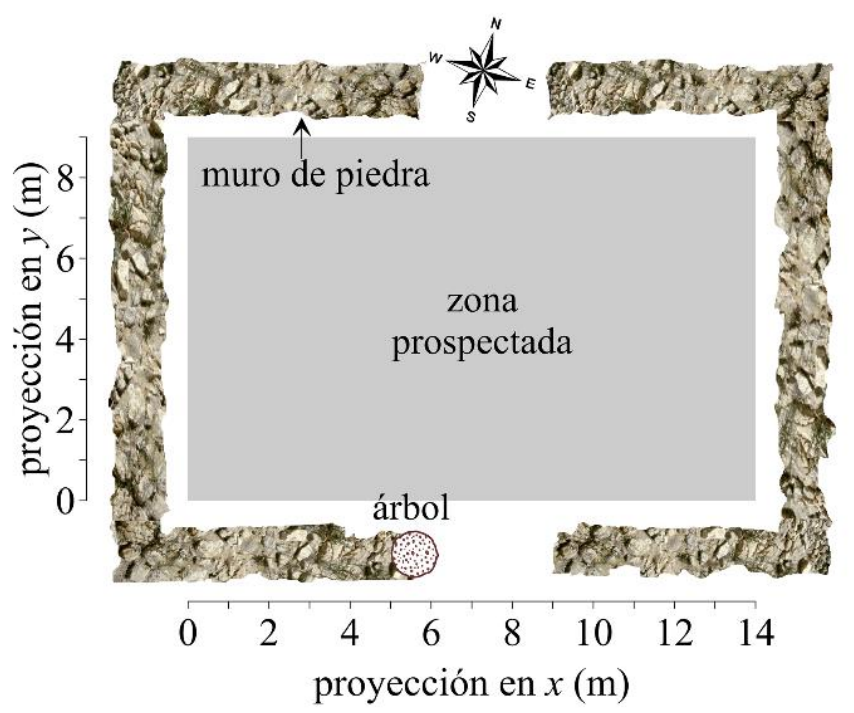

Figura 6. Puerta de Tierra, zona Sur del sitio de San Carlos de Portobelo, donde fue desarrollada la prospección electromagnética EM-38.

Las mediciones de la conductividad eléctrica aparente se realizaron cada metro a lo largo de perfiles de $14 \mathrm{~m}$ de longitud separados entre ellos una distancia de $1 \mathrm{~m}$. Los valores de este parámetro físico fueron obtenidos tanto para el dipolo horizontal como el vertical, lo que representa la medida de la conductividad eléctrica aparente del subsuelo a dos profundidades efectivas diferentes: $75 \mathrm{~cm}$ para la 
configuración dipolar horizontal y $175 \mathrm{~cm}$ para el vertical. La disposición del dispositivo EM-38 de Geonics para la medición de la conductividad eléctrica aparente del subsuelo en modo dipolo vertical y horizontal, se muestran en la Figura 7.

(a)

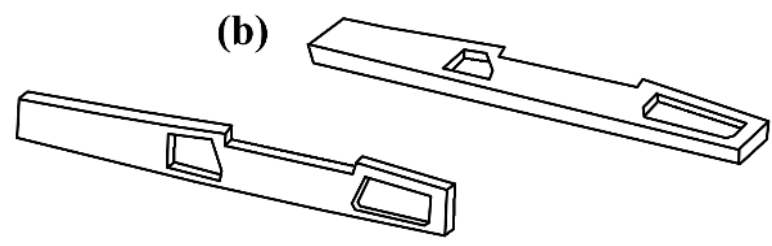

Figura 7. Disposición del dipolo del EM-38 en modo (a) vertical y (b) horizontal.

Antes de llevar a cabo la prospección electromagnética, se procedió con la fase de calibración del dispositivo; la misma consistió en obtener lecturas a $1,5 \mathrm{~m}$ de altura del suelo y llevar las mismas a 0 $\mathrm{mS} / \mathrm{m}$ (mili Siemens por metro - unidad de la conductividad eléctrica), luego se procedió en registrar nuevamente las lecturas sobre la superficie del terreno y se ajustó a 0 nuevamente de forma tal que al final, la respuesta del dipolo vertical sea el doble que la horizontal a 1,5 m de altura del suelo.

\subsection{La tomografía de resistividad eléctrica $2 \mathrm{D}$}

En todas las tomografías desarrolladas en este trabajo, se emplearon un total de 48 electrodos de acero inoxidable, separados una distancia de $50 \mathrm{~cm}$ entre ellos a lo largo de un perfil de 23,5 m de longitud. Se utilizaron además dos sistemas multi-cable de 24 conexiones cada uno con el objetivo de interconectarlos 48 electrodos. Ambos sistemas multi-cable se conectan al resistivímetro el cual cuenta con un multiplexor cuya función es la conmutar la posición de 4 electrodos a lo largo del perfil. La Figura 8 muestra la distribución de los electrodos sobre un perfil dado y el dispositivo Syscal R1 empleado en este estudio.

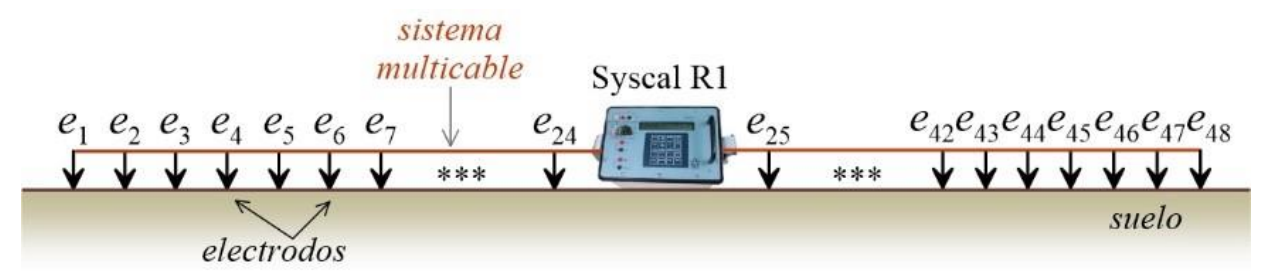

Figura 8. Distribución de los 48 electrodos sobre un determinado perfil interconectados al resistivímetro Syscal R1 de IRIS Instruments, a través de un par de sistemas multi-cable.

Anterior al trabajo de campo, el dispositivo es configurado de dos formas: la adquisición la cual consiste en programar el sistema de tal forma que, en una sola posición, se inyecte 3 veces una determinada intensidad de corriente eléctrica (3 stacks); inmediatamente, el sistema calcula un factor de calidad Q (voltaje entre corriente eléctrica) el cual representa un error estándar de estas 3 mediciones. Dicho factor Q se programó para un máximo de 1\% con estas 3 mediciones; si este valor sobrepasa el 1\%, entonces el sistema inyecta 3 veces más con el objetivo específico de mejorar la calidad de los datos de campo. También se programa el tiempo de adquisición de cada dato (500 milisegundos) y la forma de la onda cuadrada. La segunda configuración es de tipo geométrica; es decir, que se especifica la distancia entre los electrodos (50 cm como se mencionó con anterioridad), 
la configuración electródica: dipolo-dipolo o Wenner-alfa dependiendo de la zona de trabajo, el número de electrodos y el número de niveles de profundidad.

Para mejor explicar la mecánica de adquisición de datos, presentamos el diagrama de la Figura 9 el cual corresponde a una configuración electródica tipo Wenner-alfa. Los 4 electrodos se corren iniciando con los 4 primeros sobre el perfil y terminando con los 4 últimos con la secuencia $A M N B$ (para la configuración electródica tipo dipolo-dipolo, la secuencia sería $A B M N$ como se había mencionado anteriormente). El resto del proceso consiste en mantener la misma secuencia, pero inhabilitando un electrodo de por medio entre los dos pares, y así sucesivamente hasta completar los niveles de profundidad especificados en la configuración geométrica del sistema.

(a)

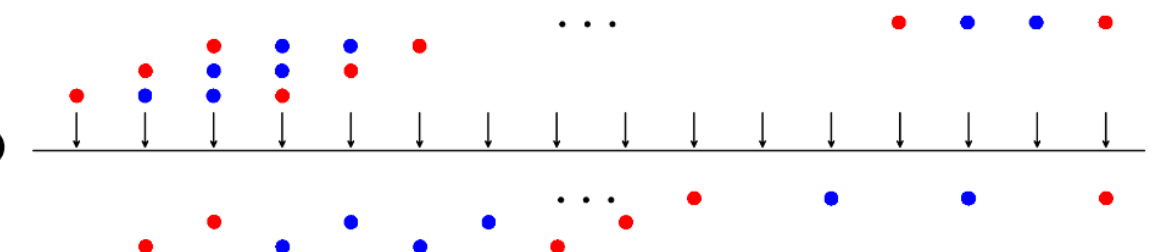

(b)

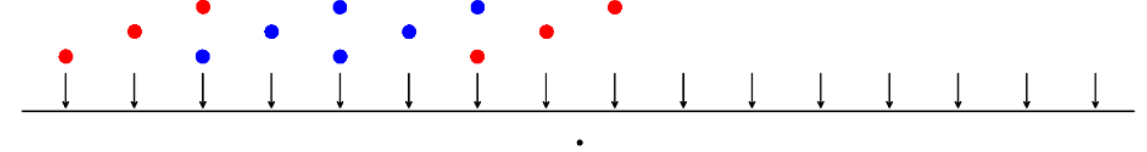

(c)

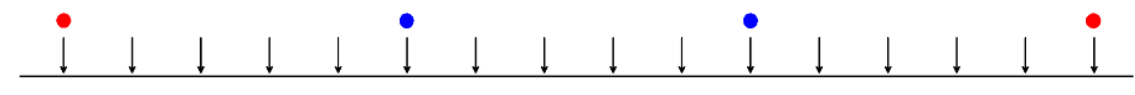

- posición de electrodos de voltaje

- posición de electrodos de corriente eléctrica

Figura 9. Arreglo Wenner-alfa y metodología de registro del voltaje para (a) el primer nivel de profundidad $n=1$, (b) segundo nivel de profundidad $n=2$, y para el último nivel de profundidad $n=5$. Las flechas representan los electrodos.

Cada conjunto de datos de resistividad eléctrica aparente medidos en campo, fue invertido empleando la norma L2 como restricción del método de mínimos cuadrados regularizado; dicha norma se encuentra implementada en el programa Res2Dinv de Geotomo Software.

\section{Resultados experimentales}

\subsection{Puerta de Tierra (sector Sur del sitio de San Carlos de Portobelo)}

Los pares de datos de conductividad eléctrica aparente medidos en campo, fueron interpolados a través de un programa Surfer 12 de Golden Software; los ma pas que muestran la distribución espacial de cada conjunto de conductividad eléctrica aparente se presentan en la Figura 10. 

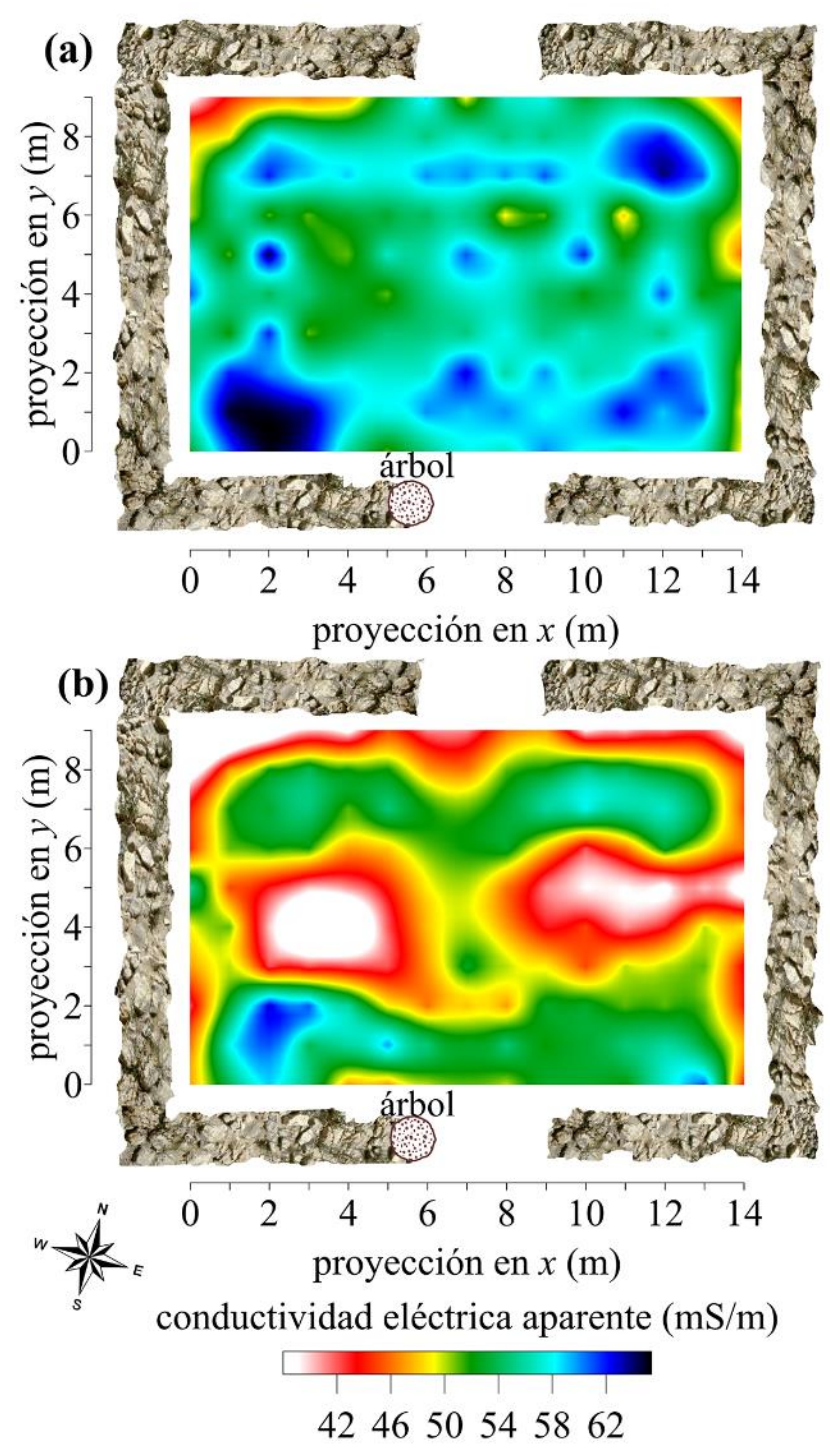

Figura 10. Mapa de conductividad eléctrica aparente del subsuelo obtenido con el dispositivo EM38 de Geonics empleando un dipolo (a) vertical $[150 \mathrm{~cm}$ de profundidad efectiva] y (b) horizontal [75 $\mathrm{cm}$ de profundidad efectiva].

En el primer mapa (Figura 10 (a)) se observan un conjunto de anomalías eléctricas de baja conductividad (en tonalidades blanco y rojo), con un rango de valores de dicho parámetro inferior a $42 \mathrm{mS} / \mathrm{m}$; dichas anomalías, que se ubican en la parte superior izquierda y derecha del mapa mencionado, se encuentran asociadas a los restos de las murallas que aun afloran en el sitio y que forman una estructura rectangular. Las anomalías en tonalidad azul que se presentan en este mapa se caracterizan por presentar valores de alta conductividad eléctrica aparente $(>58 \mathrm{mS} / \mathrm{m})$, propios de suelos con un alto contenido de humedad; las anomalías más notables se presentan en los extremos inferior izquierdo y superior derecho del mapa; sin embargo, otras anomalías de menor intensidad se pueden identificar en la parte inferior del mapa. El resto del área bajo estudio presenta una distribución bastante homogénea de la conductividad eléctrica aparente del suelo, hecho que refleja la ausencia de posibles estructuras arqueológicas a la profundidad efectiva de $150 \mathrm{~cm}$. 
Con relación al segundo mapa (Figura 10 (b)), las anomalías de baja conductividad eléctrica del subsuelo se concentran con mayor intensidad en los bordes del dicho mapa, propio de los escombros o cimientos que formaron parte de los muros y que necesitan determinarse a través de un estudio arqueológico. Pero aparte de estas anomalías representadas en tonalidades blanco y rojo $(<42 \mathrm{mS} / \mathrm{m})$, se pueden identificar dos de ellas en la parte centralizquierda y derecha del mapa; se trata de anomalías de alta resistividad eléctrica (inverso a la conductividad eléctrica del subsuelo), hecho que pudiera asociarlas a suelo firme con bajo contenido de humedad o rasgos enterrados. Las anomalías de alta conductividad eléctrica aparente que se representan en tonalidades celeste, azul y negro se encuentran asociadas a un suelo propio del sitio, pero con una considerable presencia de humedad.

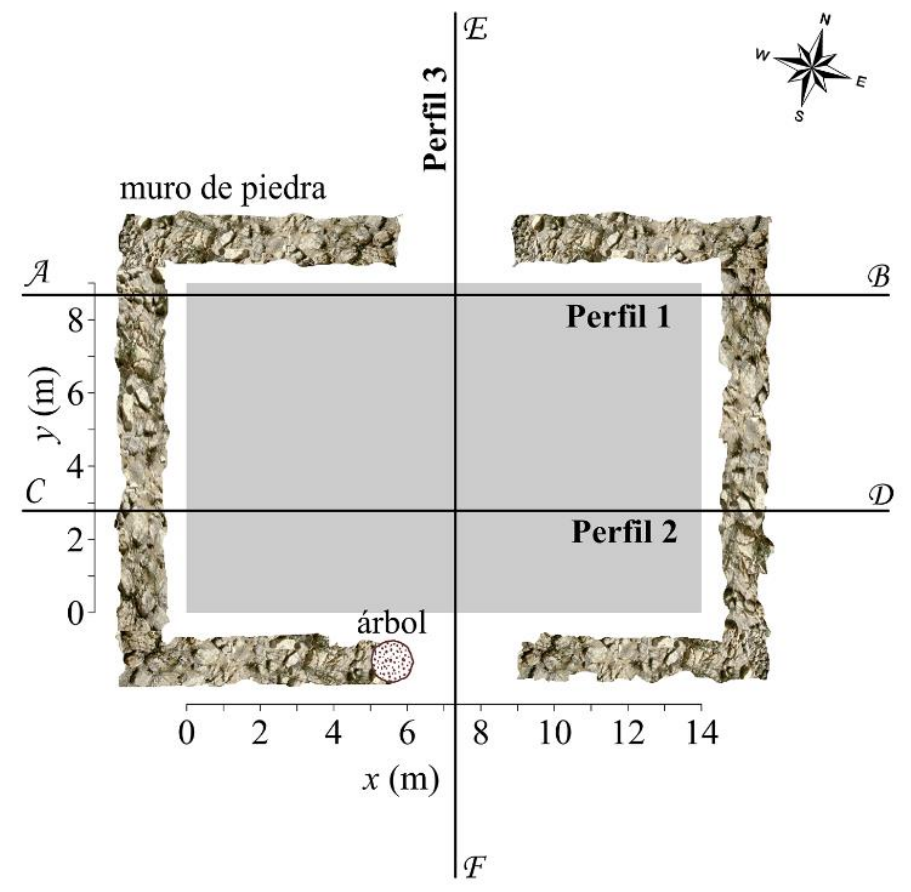

Figura 11. Distribución de los 3 perfiles eléctricos desarrollados en el área de interés.

Por otro lado, un total de 3 tomografías de resistividad eléctrica fueron desarrolladas en esta zona. La Figura 11 muestra la distribución de estas pruebas. Por otro lado, el resultado de la resolución del problema inverso para cada una de ellas se presenta en la Figura 12.

En el Perfil 1 (diagrama superior), el error de cálculo obtenido posterior a la quinta iteración fue de $0,87 \%$. Entre las posiciones 1 y $4 \mathrm{~m}$, y entre 19 y $22 \mathrm{~m}$ a lo largo del perfil, se puede identificar una fuerte anomalía eléctrica (en tonalidad fucsia), con valores de resistividad eléctrica calculada que superan los $86 \mathrm{ohm} . \mathrm{m}$; la profundidad de estas anomalías no supera los $35 \mathrm{~cm}$ y ambas se encuentran asociadas a un par de muros paralelos que afloran y que forman parte de una estructura rectangular (la Puerta de Tierra, entrada Sur del sitio de San Carlos).

Este mismo fenómeno se presenta también en la segunda tomografía, correspondiente al Perfil 2; dicho perfil (paralelo al Perfil 1 y separados entre sí una distancia de 5,75 m) fue obtenido posterior a la quinta iteración, en donde el error de cálculo fue de 1,13\%. La profundidad de estas anomalías eléctricas varía entre los 30 y $40 \mathrm{~cm}$, con el mismo rango de valores de resistividad eléctrica calculada mencionado en el Perfil 1. 

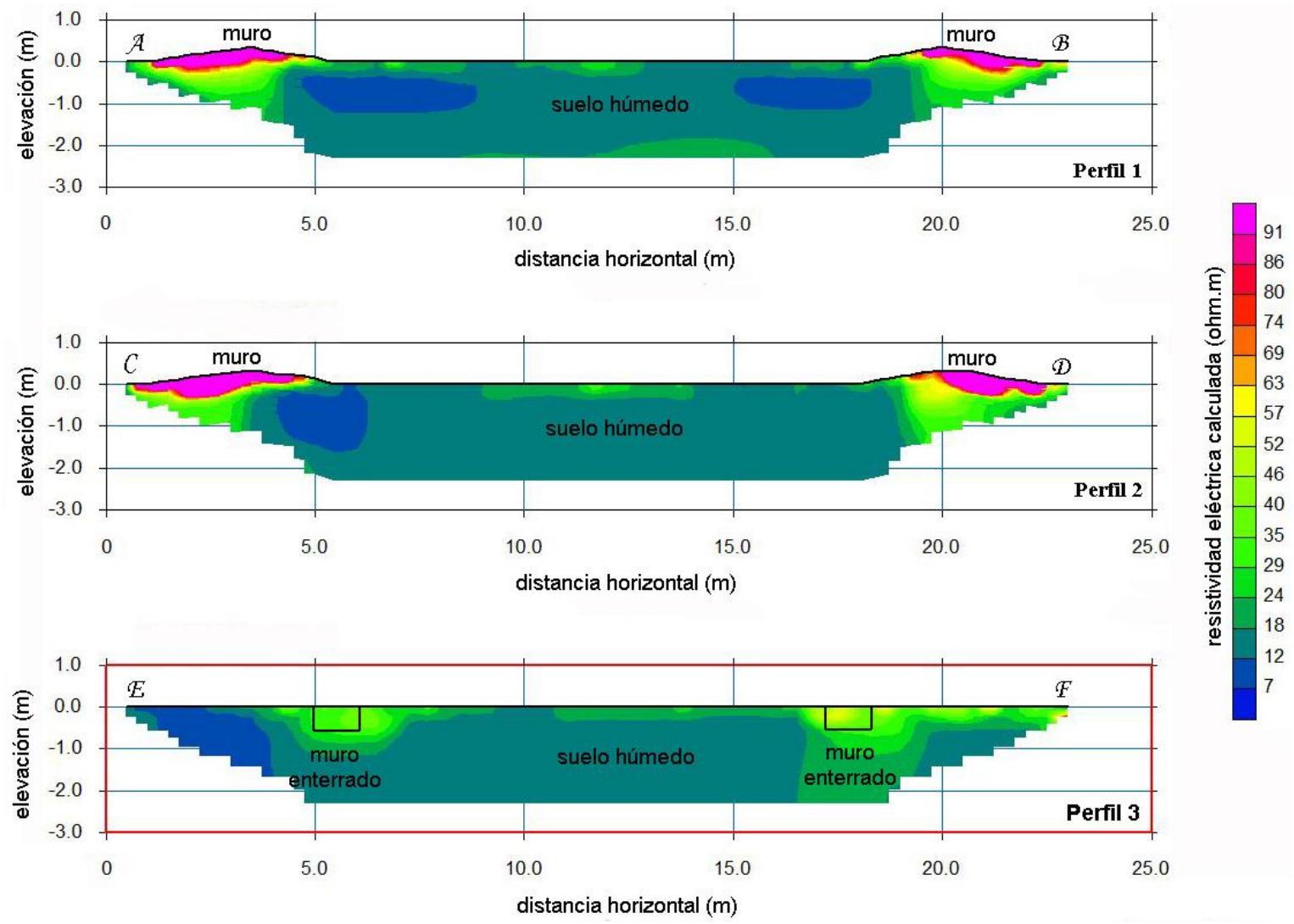

Figura 12. Tomografías de resistividad eléctrica desarrolladas en la Puerta de Tierra, sector Sur-del sitio de San Carlos de Portobelo.

En ambas tomografías (Perfiles 1 y 2), es posible identificar algunas anomalías eléctricas en tonalidad verde, y con un rango de valores de resistividad eléctrica calculada que oscila entre 24 y 40 ohm.m; estas anomalías podrían estar asociadas al tipo de suelo característico del sitio, pero con un bajo nivel de humedad, o suelo firme. Cabe destacar que estas anomalías se asocian con las identificadas en la prospección electromagnética (dipolo horizontal, con profundidad efectiva de $75 \mathrm{~cm}$ ), en donde se detectaron anomalías de baja conductividad eléctrica aparente. El resto de las anomalías eléctricas representadas en tonalidad azul y verde oscuro, con valores de resistividad eléctrica calculada que oscilan entre 7 y 24 ohm.m, se asocian con el suelo característico del sitio, pero con un elevado contenido de humedad.

La tercera tomogra fía de resistividad eléctrica (Perfil 3), fue obtenida también para una quinta iteración en donde el error de cálculo fue de $0,81 \%$; entre las posiciones 5 y $6 \mathrm{~m}$, y entre los 18,5 y 17,5 m se observan un conjunto de anomalías eléctricas en tonalidades verde y amarilla, correspondientes a valores intermedios de resistividad eléctrica calculada (24 y $63 \mathrm{ohm} . \mathrm{m})$, dichas anomalías parecen asociarse a muros enterrados (cimientos); estas anomalías llegan a profundidades que oscilan entre los 35 y $40 \mathrm{~cm}$, aproximadamente. El resto de las a nomalías en tonalidad azul y verde oscuro corresponden a suelo característico del sitio, pero con un alto contenido de humedad, tal y como se había mencionado en los resultados precedentes. 


\subsection{Baluarte de San Pedro}

En esta zona se establecieron 3 perfiles de 23,5 m de longitud cada uno al igual que las pruebas anteriores, empleándose un total de 48 electrodos en cada perfil separados una distancia de $50 \mathrm{~cm}$ entre ellos y una configuración electródica correspondiente al dipolo - dipolo. La Figura 13 presenta la distribución de estos perfiles en el Baluarte de San Pedro.

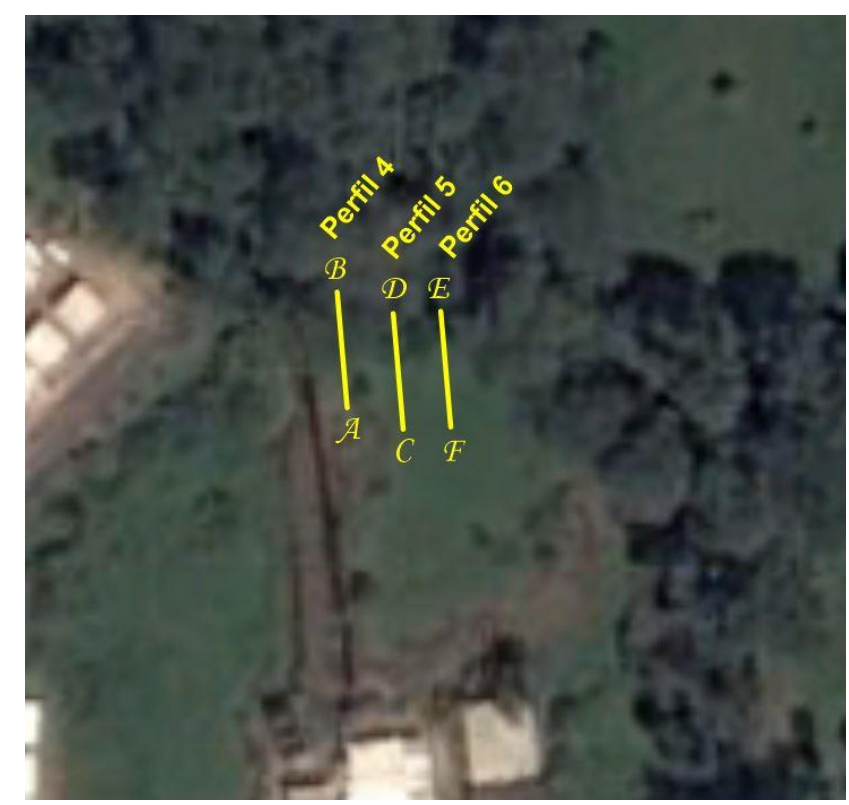

Figura 13. Distribución de los 3 perfiles eléctricos establecidos en el baluarte de San Pedro, antigua ciudad de San Carlos de Portobelo.

La resolución del problema inverso para cada uno de los perfiles establecidos en esta parte de las ruinas históricas se presenta en la Figura 14. Para el Perfil 4 (tomografía superior), el error de cálculo obtenido fue de 0,99\% para un total de 5 iteraciones. En este resultado, se pueden identificar un total de 3 fuertes anomalías de resistividad eléctrica (en tonalidad fucsia) con valores superiores a los 82 ohm.m y muy superficiales, y el primer conjunto de anomalías se ubica entre 2 y $4 \mathrm{~m}$ a lo largo del perfil y a una profundidad no máxima de $20 \mathrm{~cm}$; dichas anomalías podrían estarasociadas a escombros o rocas dispersas.

El siguiente conjunto de fuertes anomalías superficiales se encuentra ubicada entre los 6 y $9 \mathrm{~m}$ a lo largo del perfil, con profundidades que no sobrepasan los $60 \mathrm{~cm}$; dichas anomalías se encuentran asociadas a un conjunto de raíces de un árbol seco que aflora en el terreno. La tercera anomalía eléctrica de alta resistividad ( $>82 \mathrm{ohm} . \mathrm{m})$ se encuentra ubicada entre las posiciones 12,5 y 13,5 m a lo largo del perfil y la misma se asocia al muro del baluarte; es importante señalar que este perfil se estableció muy cerca del final del muro de la parte Noroeste del Baluarte de San Pedro. 

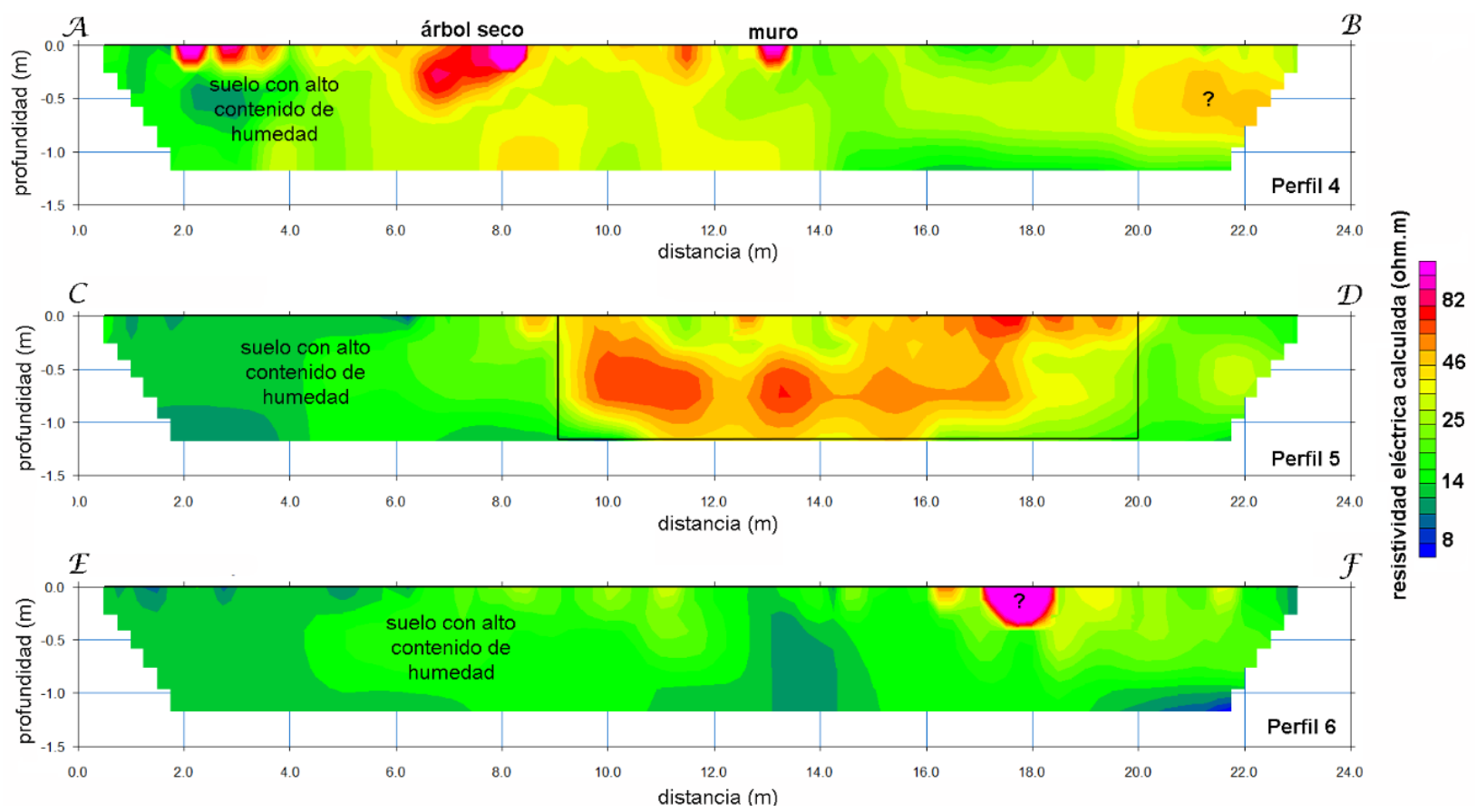

Figura 14. Tomografías de resistividad eléctrica desarrolladas en los tres perfiles establecidos en el Baluarte de San Pedro.

Entre las posiciones 20 y $22 \mathrm{~m}$ a lo largo del Perfil 4, se presenta una anomalía de resistividad eléctrica con valores moderadamente elevados (en tonalidades amarillo y naranja), con una profundidad de aproximadamente $70 \mathrm{~cm}$; es importante mencionar que después de los $16 \mathrm{~m}$ a lo largo de este perfil, la presencia de humedad y vegetación era muy notable; sin embargo, es una anomalía extensa que complica su interpretación. El resto de las anomalías eléctricas que se presentan en el sitio (anomalías en tonalidades azul y verde claro), poseen un rango de valores de resistividad eléctrica calculada que varía entre 8 y $25 \mathrm{ohm}$.m; las mismas se encuentran asociadas al tipo de suelo característicos del sitio con un elevado contenido de humedad.

Para el Perfil 5 (segunda tomografía eléctrica), el error de cálculo obtenido fue de 1,36\% para un total de 5 iteraciones. En este resultado, entre los 9 y $20 \mathrm{~m}$ a lo largo del perfil, se puede identificar un conjunto de anomalías eléctricas en tonalidades rojo y naranja, con valores de resistividad eléctrica calculada que oscilan entre 25 y $82 \mathrm{ohm} . \mathrm{m}$. Este conjunto de anomalías eléctrica podría estarasociados a la vegetación existente en el sitio (raíces, etc.), aunque a nivel superficial, el límite del rec tángulo señalado en la imagen, corresponde a la zona que no presenta humedad en superficie. Llama notablemente la atención de la profundidad de este conjunto de anomalías eléctricas $(>1 \mathrm{~m}$, aproximadamente). El resto de las anomalías eléctricas representadas en tonalidades azul y verde $(8 \rightarrow$ $25 \mathrm{ohm} . \mathrm{m})$, se asocian a suelo con notable contenido de humedad.

El Perfil 6 de la Figura 14 (tomografía inferior), el error de cálculo obtenido posterior a una quinta iteración en la resolución del problema inverso fue de $0,59 \%$. En este resultado, se puede identificar una fuerte anomalía eléctrica ubicada entre 17,0 y 18,5 $\mathrm{m}$ a lo largo del perfil. Esta anomalía posee una profundidad de aproximadamente $70 \mathrm{~cm}$ y es muy superficial; el mismo podría estar asociada a una roca o alguna raíz por la presencia de vegetación en el sitio. El resto de las anomalías eléctricas que se presentan en el tomograma (en tonalidades azul y verde), se caracteriza por tener un rango de valores 
de resistividad eléctrica calculada que varía entre 8 y $25 \mathrm{ohm} . \mathrm{m}$ (como en las pruebas anteriores); dichas anomalías eléctricas se encuentran asociadas al suelo característico del sitio con una alta presencia de humedad. En este resultado, no se presentan anomalías eléctricas asociadas a la zona de actividades de construcción mencionadas con anterioridad.

\section{Conclusiones}

En esta primera fase de estudio, podemos mencionar que la calidad de los datos obtenidos en la prospección geoeléctrica 2D, es bastante buena ya que el factor de calidad Q fue inferior a 1\%; de aquí que los errores absolutos o de cálculo obtenidos no superaran el 1,36\%. Con respecto a la Puerta de Tierra - zona Sur del sitio de San Carlos (entrada de la nueva ciudad) -, la prospección electromagnética obtenida a una profundidad de $150 \mathrm{~cm}$ (dipolo vertical - Fig. 10 (a)) no reflejó anomalías de conductividad eléctrica aparente que revelaran posibles fundaciones u otros rasgos arqueológicos enterrados a esa profundidad; sin embargo, el mapa de conductividad eléctrica aparente obtenido con el uso del dipolo horizontal (Fig. 10 (b)), muestra en los bordes del mapa, un conjunto de anomalías de valores bajos de conductividad eléctrica, lo que significa que la resistividad eléctrica es alta, característica de posibles escombros, restos de los muros o posibles cimientos que se observan en el sitio. También fue posible identificar dos principales anomalías de conductividad eléctrica aparente alineadas en la parte central del área de interés, posiblemente asociada a suelo con bajo contenido de humedad (suelo firme) o algún rasgo enterrado. Por otro lado, las tomografías de resistividad eléctrica $2 \mathrm{D}$ obtenidas en este mismo sector, logró revelar la profundidad de los muros que se encuentran en el sitio (Fig.12, Perfiles 1 y 2) y las fundaciones ubicadas en ambas entradas de la estructura o entrada a la ciudad (Fig. 12, Perfil 3). Con respecto a las tomografías de resistividad eléctrica 2D desarrolladas en el Baluarte de San Pedro, un conjunto de anomalías eléctricas superficiales pudo ser identificadas claramente, asociándose las mismas a posibles rocas, a raíces de árboles secos y a las bases de la antigua muralla (Fig. 14, Perfil 4); otras anomalías de menor intensidad se aprecian con mayor claridad en el Perfil 5 o perfil central. El resto de las anomalías eléctricas de baja resistividad eléctrica, se asocian con el suelo característico del sitio con una notable presencia de humedad.

Estos primeros resultados han expuesto situaciones puntuales de interés al proyecto en curso y que necesitarán ser profundizadas con nuevas pruebas geofísicas en las áreas estudiadas para complementar los datos ya obtenidos y aclarar con mayor atención algunas lagunas que han surgido, con la intención de evaluar la posible intervención de excavaciones arqueológicas de estudio en estos sectores. Los datos aquí descritos y obtenidos a través de la prospección geomagnética constituyen una parte del proyecto interdisciplinario de estudio sobre las estructuras del sitio de San Carlos de Portobelo, y contribuyen a orientar las siguientes etapas del proyecto. Los datos se integrarán y complementarán con los resultados de los análisis de las técnicas de construcción de la época, de los estudios históricos en archivos y de las excavaciones arqueológicas in situ para generar una panorámica integral sobre el estudio del bien histórico y de toda su extensión territorial.

\section{Agradecimientos}


Proyecto de colaboración para la Dirección Nacional de Patrimonio Histórico (DNPH) del Ministerio de Cultura de Panamá y el Patronato de Portobelo y San Lorenzo (PPSL). Se agradece a las autoridades: Dra. Katti Osorio (DNPH), Arq. Wilhelm Franqueza (PPSL), y a la participación en campo del Arq. Rodolfo Suñé (PPSL).

Al Laboratorio de Investigación en Ingeniería y Ciencias Aplicadas del Centro Experimental de Ingeniería de la Universidad Tecnológica de Panamá, por su importante colaboración e interés en este proyecto.

Al Smithsonian Tropical Research Institute, por la facilitación del equipo EM-38.

Al Archivo General de Indias del Ministerio de Cultura y Deporte de España, por la imagen MPPanamá,95 que se utiliza en este artículo para fines educativos.

\section{Bibliografia}

Bevan, B.W. 1983. Electromagnetics for mapping buried earth features. Journal of Field Archaeology, 10, $47-54$.

Dalan, R.A. 1991. Defining archaeological features with electromagnetic surveys at the Cahokia Mounds State Historic Site. Geophysics, 56(8), 1280 - 1287.

Dey, A. and Morrison, H.F. 1979. Resistivity modeling for arbitrarily shaped three - dimensional structures. Geophysics, 44 (4), $753-780$.

Frohlich, B. y Lancaster, W.J. 1985. Electromagnetic surveying in current Middle Eastern archaeology: Application and evaluation. Geophysics, 51(7), 1414 - 1425.

McNeill, J.D. 1980. Electromagneticterrain conductivity measurement at low induction numbers. Reporte Técnico TN-6, Geonics Ltd, Canadá.

Nover, G. 2005. Electrical properties of crustal and mantle rocks - a review of laboratory measurements and their explanation. Surveys in Geophysics. 26, 593 - 651.

Samouëlian, A.; Cousin, I.; Tabbagh, A.; Bruand, A. and Richard, G. 2005. Electrical resistivity survey in soil science: a review. Soil and Tillage Research. 83 (2), $173-193$.

Scollar, I.; Tabbagh, A.; Hesse, A. y Herzog, I. 1990. ArchaeologicalProspecting and Remote Sensing. Gran Bretaña: Cambridge University Press, 674 p.

Sudduth, K.A.; Drummond, S.T. y Kitchen, N.R. 2001. Accuracy issues in electromagnetic induction sensing of soil electrical conductivity for precision agriculture. Computers and Electronics in Agriculture, 31, 239-264.

Tabbagh, A. 1992. Méthodes géophysiques appliquées à la prospection archéologique. Mém.Soc. Géol. France. 161, $9-15$. 
Telford, W.M.; Geldart, L.P. y Sheriff, R.E. 1996. Applied Geophysics. EEUU: Cambridge University Press, $770 \mathrm{p}$.

Wynn, J.C. 1986. A review of geophysical methods used in archaeology. Geoarchaeology, 1(3), 245 - 257. 